\title{
Appendix
}

\section{LIST OF INTERVIEW LOCATIONS}

\section{South Sudan}

Aweil, Northern Bahr El Ghazal

Bentiu POC and Bentiu town, Unity

Bor, Jonglei

Ding Ding, Unity

Juba, Central Equatoria

Kajo Keji, Central Equatoria

Kerua, Central Equatoria

Koch town, Unity

Lankien, Jonglei

Lanya, Central Equatoria

Logo, Central Equatoria

Malakal, Upper Nile

Morobo, Central Equatoria

Nimule, Eastern Equatoria

Nyal, Unity

Rumbek, Lakes

Torit, Eastern Equatoria

Wau, Western Bahr El Ghazal

Yei, Central Equatoria

Yuai, Jonglei 


\section{Uganda}

Bidi Bidi refugee camp

Imvepi refugee camp

Kampala

Lamwo refugee camp

Rhino refugee camp

Yumbe

\section{Ethiopia}

Addis Ababa

\section{Kenya}

Nairobi

\section{USA}

New York

Washington, DC

\section{France}

Paris 